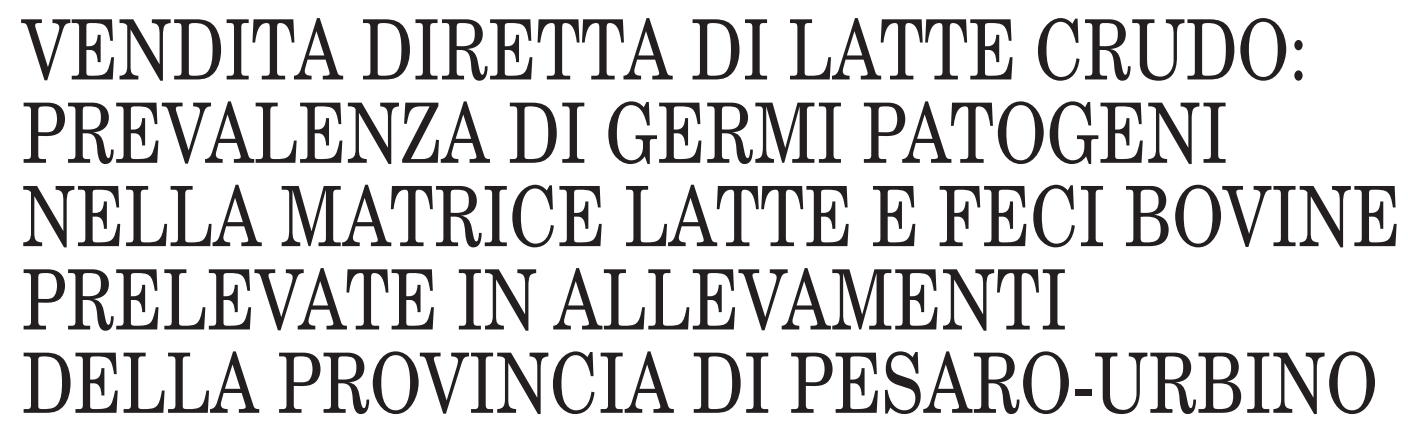

\title{
THE DIRECT SALE OF RAW MILK: PREVALENCE OF PATHOGENS IN RAW MILK AND BOVINE FAECES COLLECTED IN FARMS WITHIN THE PROVINCE OF PESARO-URBINO
}

\author{
Petruzzelli A., Sola D., Paolini F., Baldassarri S., Orazietti N., \\ Foglini M., Micci E., Pezzotti G., Cenci T., Tonucci F. \\ Istituto Zooprofilattico Sperimentale dell'Umbria e delle Marche
}

SUMMARY

Key words

\begin{abstract}
In the last years, direct sale of raw milk by vending machines has largely increased in several European Countries and Italy. As a consequence, adequate hygienic measures and correct consumer's information is required in order to reduce any potential risk linked to this product. In the present study, the occurrence of pathogens (Salmonella spp., verocytotoxigenic E.coli, Campylobacter spp., Listeria monocytogenes, S.aureus) in raw milk and bovine faeces collected in 4 farms in the province of Pesaro-Urbino (Italy), between January 2007 and March 2009 has been investigated; 99.5\% of milk samples resulted negative for the pathogens considered and complying with the regulation $S$. aureus limits. Campylobacter has been found in $0.44 \%$ of the samples, collected during summer, while only one sample resulted positive to a non-verocytotoxigenic E.coli O157. In respect to faeces, $62.6 \%$ of the samples resulted negative, $33.6 \%$ were contaminated by Campylobacter spp. (68\% Campylobacter coli and $32 \%$ Campylobacter jejuni) and $3.8 \%$ by E.coli O157. No samples resulted positive for Salmonella spp. or Listeria monocytogenes. The results highlight the necessity of a strict plan of hygienic and sanitary controls, with particular attention to milking process hygiene and raw milk storage, to reduce the risk of contamination of the product.
\end{abstract}

Milk snack's, bovine faeces, raw milk
Negli ultimi anni si è assistito, in Europa (Austria, Svizzera, Germania) ed attualmente anche in molte regioni italiane, alla vendita diretta di latte crudo attraverso distributori automatici (milk snack's). Le caratteristiche di tale prodotto, le modalità di distribuzione e di consumo evidenziano la necessità di mantenere un elevato livello di sorveglianza igienico sanitaria e di fornire al consumatore un'adeguata informazione circa i pericoli legati al consumo di tale alimento, i tempi e le modalità di conservazione. La normativa comunitaria (Regolamento 853/2004/CE) consente la commercializzazione di latte crudo direttamente al consumatore solo se il produttore predispone un piano di auto- controllo che garantisca la salubrità dell'alimento. In Italia, la sorveglianza sui rischi connessi alla vendita diretta di latte crudo, è regolamentata dall'Intesa Stato Regioni del 25 gennaio 2007 recepita dalla regione Marche attraverso il Decreto Dirigenziale n. 123 del 26 maggio 2008. Inoltre il 10 dicembre 2008 è stata emanata un'Ordinanza del Ministero del Lavoro in cui si evidenzia l'obbligo di apporre sulle macchine erogatrici la dicitura "prodotto da consumarsi dopo bollitura" e di indicare 3 giorni come data di scadenza del prodotto dal momento del prelievo dal distributore. Quest'ultima normativa è stata emanata in conseguenza di alcune segnalazioni pervenute dall'Istituto Superiore di Sanità 
(Nota del 22 ottobre 2008 della Direzione Generale della Sicurezza alimentare del Ministero, le Regioni e Province Autonome) relativa a dieci casi di Sindrome Uremica Emolitica provocata dal germe E. coli verocitotossico in bambini che avevano consumato latte crudo. Pur non essendo dimostrata una correlazione di tali focolai epidemici con il consumo diretto di tale alimento, non potendo escludere il rischio di contaminazione da patogeni fecali e ambientali, il Ministero ha ritenuto opportuno adottare misure preventive restrittive.

In questo lavoro vengono presentati i risultati relativi alla ricerca di germi patogeni (Salmonella spp., E. coli verocitotossico, Campylobacter termotolleranti, Listeria monocytogenes, S. aureus) in matrici di latte bovino prelevate in autocontrollo in 4 allevamenti della provincia di Pesaro-Urbino nel periodo gennaio 2007-marzo 2009 e in pool di feci prelevati nello stesso periodo in soli 2 dei precedenti allevamenti.

Sono stati analizzati complessivamente 1469 campioni di latte crudo (804 nell'anno 2007, 579 nell'anno 2008 e 86 da gennaio a marzo 2009) per la ricerca di tutti i germi patogeni previsti dall'Intesa Stato Regioni e 131 pool di feci, costituiti da 5 campioni corrispondenti ad ogni singola bovina, (69 nell'anno 2007 e 62 nell'anno 2008) per la ricerca di Salmonella spp., E. coli verocitotossico, Campylobacter termotolleranti, Listeria monocytogenes. I campioni di latte sono stati analizzati con il metodo ELFA (Enzyme Linked Fluorescent Assay) per la ricerca di germi patogeni e metodo colturale per la numerazione dello $S$. aureus mentre i pool di feci sono stati analizzati con metodi colturali.

Il 99.5\% (1461 campioni) dei campioni di latte crudo analizzati sono risultati negativi per la presenza di germi patogeni e conformi ai limiti di legge per $S$. aureus, mentre lo $0.44 \%$ ( 7 campioni) sono risultati positivi per la presenza di Campylobacter termotollerante e lo $0.06 \%$ ( 1 campione) per la presenza di $E$. coli $\mathrm{O} 157$. Il ceppo di $E$. coli $\mathrm{O} 157$ isolato è risultato non verocitotossico in quanto negativo al saggio di tossicità su cellule Vero e alla PCR per la presenza dei geni codificanti VT1 e VT2.

Relativamente ai pool di feci, il $62.6 \%$ (82 pool) di campioni analizzati sono risultati negativi per germi patogeni mentre il 33.6\% (44 pool) sono risultati positivi per Campylobacter termotollerante con una prevalenza del $68 \%$ di Campylobacter coli e $32 \%$ di Campylobacter jejuni infine il 3.8\% (5 pool) dei campioni sono risultati positivi per E. coli O157 non verocitotossico. In particolare la presenza di quest'ultimo patogeno è stata rilevata in pool di feci campionati in un unica seduta di prelievo effettuata in un unico allevamento. Tutti i campioni di feci sono risultati negativi per Salmonella spp. e Listeria monocytogenes.

Dai risultati ottenuti si evidenzia la necessità di un rigoroso rispetto delle idonee procedure di gestione della fase di mungitura da parte dell'operatore in quanto la prevalenza di germi patogeni, rilevata nelle feci bovine, potrebbe provocare la contaminazione della matrice latte. Inoltre, l'isolamento nel periodo estivo, di tali germi dal latte, suggerisce un rigoroso rispetto delle temperature di refrigerazione e una maggiore frequenza di campionamento in tale periodo risultato più suscettibile e a rischio di contaminazione.

\section{BIBLIOGRAFIA}

Aminul Islam M., Heuvelink Annet E., Talukder Kaisar, De Boer Enne. 2006. Immunoconcentration of Shiga toxinproducing $E$. coli 0157 from animal faeces and raw meats by using Dynabeads anti-E. coli 0157 and the VIDAS system. International Journal of Food Microbiology, 151-156. Decreto Dirigenziale della regione Marche n. 123 del 26 maggio 2008. Recepimento dell'Intesa, ai sensi dell'art. 8, comma 6, della legge 5 giugno 2003, n. 131, tra Governo, le Regioni e le province Autonome, n. 5/CSR del 25 gennaio 2007 in materia di vendita diretta di latte crudo per l'alimentazione umana. Intesa, ai sensi dell'art. 8, comma 6, della legge 5 giugno 2003, n. 131, tra Governo, le Regioni e le province Autonome di Trento e di Bolzano $\mathrm{n}$. 5/CSR del 25 gennaio 2007 in materia di vendita diretta di latte crudo per l'alimentazione umana. Regolamento europeo CE 853/2004- norme specifiche in materia di igiene per gli alimenti di origine animale. Ordinanza ministeriale del 10 dicembre 2008. Misure urgenti in materia di produzione, commercializzazione. 